\title{
PERFORMANCE COMPARISON OF CODED AND UNCODED IEEE 802.16D SYSTEMSUNDER STANFORD UNIVERSITY INTERIM (SUI)CHANNELS
}

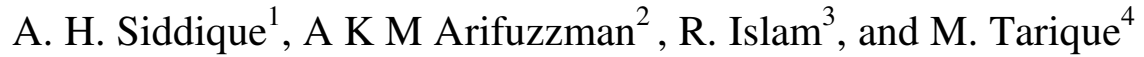 \\ ${ }^{1}$ Department of Electrical Engineering, The Petroleum Institute, Abu Dhabi, UAE \\ ${ }^{2}$ Department of ECE, University of Alabama, Birmingham, AL, USA \\ ${ }^{3}$ Department of EEE, American International University, Dhaka, Bangladesh, \\ ${ }^{4}$ Ajman University of Science and Technology, Fujairah, UAE
}

\begin{abstract}
Worldwide Interoperability for Microwave Access (WiMax), standardized asIEEE 802.16d is a popular technology for broadband wireless communication system. Orthogonal Frequency Division Multiplexing (OFDM) is the core of this technology.OFDMreduces Inter-symbol Interference (ISI) and hence improves system performance (i.e., Bit Error Rate (BER)). To improve system performance further error correction coding schemes have been included in WiMax. It is widely accepted thata coded system outperforms an uncodedsystem. But, the performance improvement of a coded system depends on the channel conditions. In this paper, we investigated and compared the performances of a coded and an uncoded WiMaxsystem under a practical channel model called Stanford University Interim (SUI). Different modulation schemes namely BPSK, QPSK, 16-QAM, and 64-QAM have been considered in this work. It is shown that the selection of codedoruncoded WiMaxsystem should depend on the channel condition as well as on the modulation used. It is also shown that anuncoded system outperforms a coded system under some channel conditions.
\end{abstract}

\section{KEYWORDS}

WiMax, IEEE 802.16d, OFDM, BER, BPSK, QPSK, 16-QAM, 64-QAM, SUI-1, SUI-2, SUI-3, SUI4,SUI-5,SUI-6.

\section{INTRODUCTION}

Broadband Wireless Access (BWA) network has been a very popular technology for the last few years. Many telecommunication equipment manufacturers offered products for BWA. Hence, there was a need for an interoperable standard. The U.S National Institute of Standards and Technology (NIST) formed the 802.16 Working Group to develop such an interoperable standard. This group introduced the IEEE 802.16 standard and defined the Physical (PHY) and Medium Access Control (MAC) layer specifications for BWA. The standard adoptedBinary Phase Shift Keying (BPSK), Quadriphase Shift Keying (QPSK), 16-QAM, and 64-QAM modulation schemes. This standard was initially introduced by assuming a Line-of-Sight (LOS) radio propagation between a transmitter and a receiver attached with an outdoor Customer Premises Equipment (CPE).This standard was then modified to correct some of the errors and inconsistencies of its previous version. According to this modified standard non Line-of-Sight (NLOS) radio propagation became possible due to its operation at frequencies that are below 11 
International Journal of Computer Networks \& Communications (IJCNC) Vol.6, No.2, March 2014

GHz. Hence, the geographical reach of the network was extended.But, multipath signalpropagation became an issuedue to the NLOS propagation. Multipath signal propagation caused high Inter-symbol Interference (ISI). To reduce ISI advanced power management and adaptive antenna arrays were included in thisstandard. In addition, a multi-carrier modulation technique named Orthogonal Frequency Division Multiplexing (OFDM) was includedand two subsequent standards were introduced namely IEEE 802.16a and IEE 802.16c. These two standards were consolidated to a new standard calledIEEE 802.16d. Thisstandard defines a network that resembles to a cellular phone network. In this network each cell consists of a Base Station (BS) and one or more subscriber station (SS) depending on the topology. The BS provides connectivity to a core network. The SS can be a roof mounted or wall mounted CPE or a standalone hand held device like mobile phone, Personal Digital Assistant (PDA) or Peripheral Component Interconnect (PCI) card for PC or Laptop.A comparison of different standards related to IEEE 802.16 are summarized and compared in Table 1.

Table 1: Comparisons of different IEEE 802.16 standards

\begin{tabular}{|l|l|l|l|l|}
\hline Specifications & IEEE 802.16(2001) & $\begin{array}{l}\text { IEEE 802.16a } \\
(2003)\end{array}$ & $\begin{array}{l}\text { IEEE 802.16 } \\
(2004)\end{array}$ & $\begin{array}{l}\text { IEEE } \\
802.16 \mathrm{e}(2005)\end{array}$ \\
\hline Spectrum & $10-66 \mathrm{GHz}$ & $2-11 \mathrm{GHz}$ & $2-11 \mathrm{GHz}$ & $2-6 \mathrm{GHz}$ \\
\hline Propagation model & Line of Sight & Non Line of Sight & $\begin{array}{l}\text { Non Line of } \\
\text { Sight }\end{array}$ & $\begin{array}{l}\text { Non Line of } \\
\text { Sight }\end{array}$ \\
\hline Bit Rate & $134 \mathrm{Mbps}$ & $75 \mathrm{Mbps}$ & $75 \mathrm{Mbps}$ & $15 \mathrm{Mbps}$ \\
\hline Bandwidth & $28 \mathrm{MHz}$ & $20 \mathrm{MHz}$ & $20 \mathrm{MHz}$ & $5 \mathrm{MHz}$ \\
\hline Modulation & QPSK, & BPSK, & OFDM, & OFDMA, \\
& 16-QAM, & QPSK, & BPSK, & QPSK, \\
& 64-QAM & 6-QAM,, & 16-QAM, \\
& & 64-QAM & $\begin{array}{l}\text { 64-QAM, }, \\
\text { 256-QAM },\end{array}$ & $\begin{array}{l}\text { 256-QAM } \\
\end{array}$ \\
& & & Fixed/Nomadic & Portable/mobile \\
\hline
\end{tabular}

Worldwide Interoperability for Microwave Access (WiMAX) forum is an alliance of telecommunication equipment manufacturers and service providersformed to promote and certify the compatibility and interoperability of BWA products employing the IEEE 802.16d.Among different physical layer specifications as shown in Table 1 OFDM based physical layer is favored by the WiMax forum. In addition to minimal Inter Symbol Interference (ISI) the other advantages of this standard include (a) flexible channel bandwidth, (b) error correction coding, and (c) adaptive modulation. The channel bandwidth can be an integer multiple of $1.25 \mathrm{MHz}$ (i.e., 1.5 $\mathrm{MHz}, 1.75 \mathrm{MHz}, 2 \mathrm{MHz}$ and $2.75 \mathrm{MHz}$ ). But, the maximum limit of a channel bandwidth is set to $20 \mathrm{MHz}$. This flexible channel bandwidth makes this technology an interoperable one. In order to improve system performance (i.e., BER) further forward error control (FEC) mechanisms have been included. The FEC is done in two phases namely (i) Reed-Solomon (RS) coding, and (ii) a convolutional coding (CC). The RS coding corrects the burst errors and the $\mathrm{CC}$ corrects the bit errors. The Turbo coding has been kept as an optional feature. The FEC and the modulations are paired to make WiMax an adaptive modulation and coding system. Some of the combinations of coding and modulation are listed in Table 2.In this paper we investigated and compared the performances of IEEE $802.16 \mathrm{~d}$ system. We considered two cases namely (i) coded system, and (ii) uncoded system. The main objective is to investigate the pros and cons of a coded system compared to those of an uncodedWiMaxsystem. In this investigation we also included Stanford University Interim (SUI) channel model. 
International Journal of Computer Networks \& Communications (IJCNC) Vol.6, No.2, March 2014

Table 2: Coded and Uncoded systems

\begin{tabular}{|l|l|l|l|l|}
\hline Modulation & $\begin{array}{l}\text { Uncoded Block } \\
\text { Size (bytes) }\end{array}$ & $\begin{array}{l}\text { Code Block size } \\
\text { (bytes) }\end{array}$ & $\begin{array}{l}\text { Overall Coding } \\
\text { rate }\end{array}$ & \\
\hline BPSK & 12 & 24 & $1 / 2$ \\
\hline QPSK & 24 & 48 & $1 / 2$ \\
\hline 16-QAM & 48 & 96 & $1 / 2$ \\
\hline 64-QAM & 96 & 144 & $2 / 3$ & \\
\hline
\end{tabular}

There have been numerous channel models proposed for wireless communication systems. These channel models can be broadly classified as (i) Indoor channel models, and (ii) Outdoor channel models [1-2]. The UWV, Saleh-Valenzeula, and IEEE 802.11 channel models are some of the examples of indoor channel models. Filtered White Gaussian Noise (FWGN), Clarke-Gans, Modified Frequency Domain FWGN, Time Domain FWGN, Jakes model, Ray based channel, Frequency Selective channel, and Stanford University Interim (SUI) channel models are the examples of outdoor channel models. In this investigation we chose SUI channel model because of its unique characteristics. Some of the unique characteristics are as follows: (a) it has a higher path loss as compared to super cell architecture, (b) it considers both macroscopic and microscopic fading effects, (c) it considers both co-channel and adjacent channel interference, and (d) it takes account of high multipath delay and Doppler spread [3]. The SUI channel also includesmany diversified parameters such as terrain, antenna specifications, wind speed, traffic range, and bandwidth. The $K$ factor is a very important aspect of this channel model. The $K$ factor depends upon BTS and CPE heights, bandwidth, distance from the antenna, and environmental conditions (i.e., wind, traffic, and season). The $K$ factor for all these 6 channels namely SUI-1, SUI-2, SUI-3, SUI-4, SUI-5, and SUI-6 are very different [3].

The rest of this paper is organized as follows: Section II briefly explains the effects of coding on the performance of a general digital communication system. Section III contains some works that are related to this work. A comprehensive description of SUI channel model has been presented in section IV. The simulation model and the simulation results have been presented in sectionV. This paper is concluded with section VI.

\section{CODED VS UNCODED SYSTEM}

To better withstand the effect of various channel impairments, such as noise, interference, and fading a coded communication system is preferred to an uncoded communication system. Coding causes various system trade-offs. Figure 1 compares the bit-error rate (denoted as $\mathrm{P}_{\mathrm{b}}$ )variation with the signal-to-noise ratio (denoted as $\mathrm{E}_{\mathrm{b}} / \mathrm{N}_{0}$ ) of typical coded and uncoded communication systems [13]. Numerous works can be found in the literatures that compared the performances of coded and uncoded communication systems. These works show that there are trade-offs that can be achieved with the use of a coded system. Some of them include (i) error performance versus bandwidth, (ii) power versus bandwidth, and (iii) data rate versus bandwidth.The error performance and bandwidth trade-off is illustrated by operating points $\mathrm{A}$ and $\mathrm{C}$ shown in Figure 1. Let us assume that a communication system has been designed to operate at point A. But, the system needsa lower bit-error rate denoted by the operating point $\mathrm{B}$. This operating point can be achieved by increasing the $\mathrm{E}_{\mathrm{b}} / \mathrm{N}_{\mathrm{o}}$ to $12 \mathrm{~dB}$. An alternative way to achieve this low bit error rate (without increasing the $\mathrm{E}_{b} / \mathrm{N}_{\mathrm{o}}$ ) is by incorporating the coding with the system. It is depicted in this figure that the system can move from operating point $\mathrm{A}$ to operating point $\mathrm{C}$ (without increasing $\mathrm{E}_{\mathrm{b}} / \mathrm{N}_{\mathrm{o}}$ ) by using coding. The power versus bandwidth trade-off can be illustrated by using operating points $\mathrm{D}$ and $\mathrm{E}$. Let us assume that a communication system has been designed to operate at point $\mathrm{D}$. This operating point provides a very low bit-error rate $\left(\right.$ i.e., $10^{-6}$ ), but it requires a very high power (i.e., $14 \mathrm{~dB}$ ). The coded system can provide this low bit-error rate at low power 
level as the operating point is shifted from D to E.In order to explain the data rate versus bandwidth trade-off let us assume that a communication system is operating at point $\mathrm{D}$. This operating point provides a very low bit error-rate at $\mathrm{E}_{\mathrm{b}} / \mathrm{N}_{\mathrm{o}}=14 \mathrm{~dB}$. The system performance is satisfactory with this low bit-error rate and it does not have any problem with the high power (i.e., $\mathrm{E}_{\mathrm{b}} / \mathrm{N}_{\mathrm{o}}$ ). The only problem is that a higher data rate is required. This can be achieved based on the following well-known relationship [13]:

$$
\frac{E_{b}}{N_{o}}=\frac{P_{r}}{N_{0}}\left(\frac{1}{R}\right)
$$

,where $\mathrm{E}_{\mathrm{b}} / \mathrm{N}_{\mathrm{o}}$ is the Signal-to-Noise Ratio (SNR), $\mathrm{P}_{\mathrm{r}}$ is the received power, and $\mathrm{R}$ is the data rate.This important relation shows that an increase in bit rate will reduce the SNR. Hence, the operating point will move from $\mathrm{D}$ to $\mathrm{F}$ (see Figure 1). This operating point represents a high bit error rate and hence there will be degradation in the quality of data. The coded system can solve this problem in the following way. If the operating point is shifted from $\mathrm{F}$ to $\mathrm{E}$ by using coding as shown in Figure 1, the bit error rate will again decrease to a low value (i.e., $10^{-6}$ ).

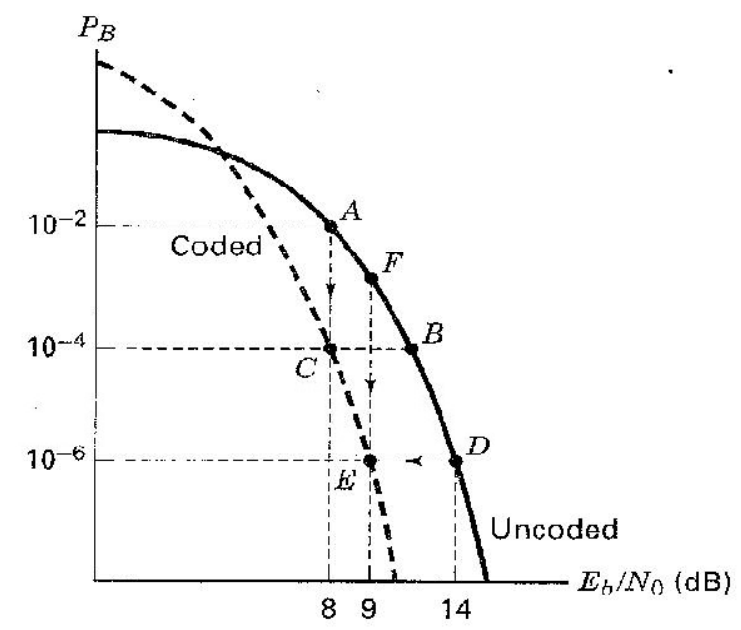

Figure 1: Comparison of performances of coded and uncoded communication systems [15]

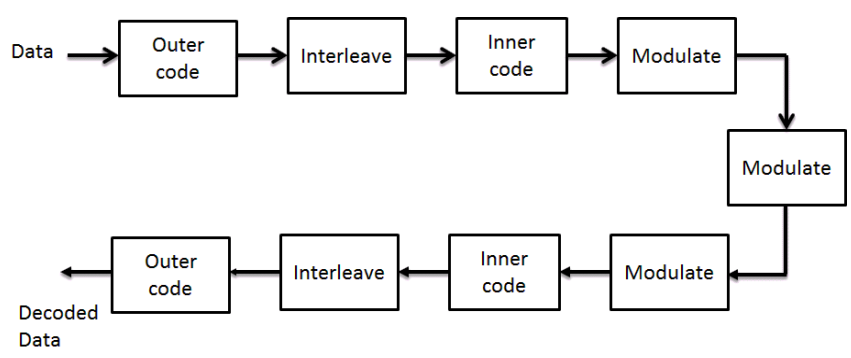

Figure 2: Concatenated coding system

It is obvious from the above mentioned trade-off examples that a coded communication system has always some advantages over an uncoded communication system. In most of the practical communication systems a concatenated coding system is used to achieve a desired performance. This type of concatenated coding scheme is shown in Figure 2. The main reason of using the concatenated coding scheme is to achieve a low bit error rate with less complexity. Otherwise, 
achieving the same low bit error rate by using a single coding scheme will be complex and it will be hard to implement in hardware. In concatenated coding schemes two levels of coding are used namely inner coding and outer coding. The inner coding interfaces modulation/demodulation and the channel. Most of the channel errors are corrected by this inner code. The outer coding further reduces the error to a specified low level.

In the physical layer of the IEEE 802.16d, the channel coding is done in three steps namely (i) data randomization, (ii) Forward Error Correction (FEC), and (iii) interleaving. The FEC is done in two phases: the outer phase is implemented by using a Reed-Solomon (RS) coder and the inner phase is implemented by a convolutional coder. The channel coding and decoding methods of this standard are shown in Figure 3.

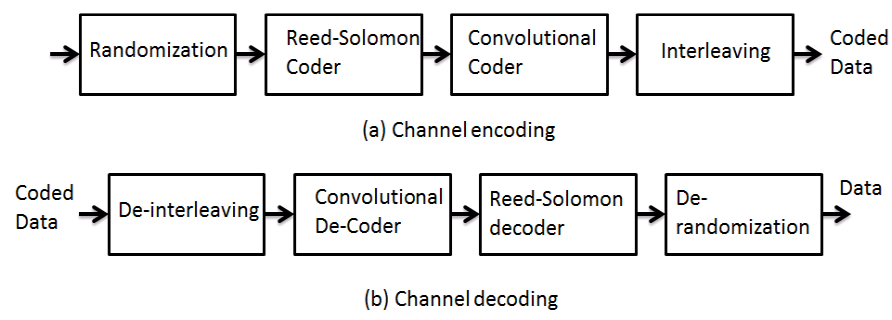

Figure 3: Channel coding and decoding in IEEE 802.16d.

The input data is randomized by using a scrambler to avoid long sequence of ones. The ReedSolomon encoder supports shortened and punctured code to facilitate variable block sizes and variable error-correction capability. The output of Reed-Solomon coded block is fed to an inner binary convolutional encoder. This encoder has a rate of $1 / 2$ and the generator polynomials are defined as follows:

$\mathrm{G}_{1}=171_{\mathrm{OCT}}$

$\mathrm{G}_{2}=133_{\mathrm{OCT}}$

The encoded data are then interleaved by a block interleaver. The size of the block interleaver depends on the number of sub-carriers used in one OFDM symbol. The interleaver has two step permutations. The first permutation maps the adjacent bits into non-adjacent sub-carriers. The second permutation ensures that the adjacent bits are mapped alternately into less or more significant bits to avoid long run of unreliable bits.

\section{RELATED WORKS}

The performance of IEEE 802.16d based Wireless MAN system has been investigated in many works since the introduction of this standard. One of the early works has been reported in [14]. In this paper a study of an iterative joint algorithm for frequency offset and time offset synchronization has been used in 256 OFDM system. It has been claimed that the IEEE 802.16d wireless-MAN systemrequires a robust synchronization mechanism. A timedomain preamble structure has been provided to detect and correct the synchronization errors. The authors claimed that this type of synchronization is not enough and they proposed a new iterative algorithm. The simulation results show that the proposed algorithm reduces the probability of synchronization failure and hence it improves the system performance. The 
performance of IEEE 802.16d under mobility condition has been investigated in [15]. Two main issues have been addressed therein namely connection handoff and correct reception of signal.The authors have proposed algorithms for seamless connection handoff.The results show that the bit error rate for the link can provide satisfactory error performance for terminal speed up to tens of kph. It is also shown that the current IEEE 802.16d standard with the proposed technique can support user mobility.Another early work that provides the insight of the IEEE 802.16d system is [16]. This work presents the analysis of realistic attainable throughput and performance of the IEEE 802.16dstandard.The authors also suggested some future enhancements to the standard so that the achievable data rate can be increased with moderate system complexity. In IEEE 802.16d standard one transmission antenna uplink based cooperative spatial multiplexing (CSM) has been adopted.But, in [17] multiple transmission antennas have been considered for mobile stations for uplink transmission. The authors have proposed a codebook based uplink transmission and compared its performances with that of CSM based system. The authors have shown that the proposed scheme results in substantial gain over the CSM in terms of throughput.Theoretical performance bound of the IEEE 802.16d channel has been examined in [18]. Different design techniques like OFDM and single-carrier frequency-domain equalization (SC-FDE), time-domain decision feedback equalization (DFE), and sphere decoder (SD) techniques have been considered and compared in the same work. The performances of uncoded IEEE 802.16d have also been investigated in [19] too. The effects of channel estimation on the system performance have been investigated in this paper and some novel channel estimation technique hasalso been proposed. The channel estimation is based on the principle of pilotsymbol-aided channel estimation (PACE) in frequency domain. The authors have proposed a simple method to reduce the computational complexity of MMSE algorithm. Theyhave presented a rotated quasi-orthogonal space-time block codes (RQOSTBC) scheme for OFDM communication based on IEEE 802.16d. At the transmitter, the data streams for the OFDM tones enter separate QOSTBC coders whose outputs are then forwarded to the different antennas. At the receiver, the pairwise decoding was employed. The SUI channel model, which is recommended by the IEEE 802.16 working group is used in the simulations to compare performance ofthe STBC and QOSTBC. In [20] a rotated quasi-orthogonal space-time block codes (RQOSTBC) scheme has been proposed for IEEE 802.16d. The data streams for the sub-carriers are sent to separate QOSTBC coders before forwarded to different antennas in the transmitter side and the pairwise decoding is employed in the receiver side. The SUI channel models have been used in the simulations to compare the performances of the STBC and QOSTBC. Although OFDM has been used in IEEE $802.16 \mathrm{~d}$ system, some works have considered other modulations. For example, multi-carrier CDMA has been used in [21] as a transmission scheme instead of OFDM. The authors have investigated the performance of MC-CDMA based system with that of OFDM based system. Different modulation and forward error correction (FEC) coding methods have been considered. Walsh-Hadamard code has been used as spreading code. It has been shown that the WiMAX system based on MC-CDMA outperforms the conventional OFDM.

One of the early works investigating the performances of IEEE 802.16 based on some experimental results has been reported in [22]. The experiments were conducted based on a deployed IEEE 802.16d network at Clemson University. The frequency of operation of the deployed system was $4.9 \mathrm{GHz}$. The authors have claimed that more realistic assumptions must be used in order to accurately model and analyze WiMAX. The authors also compared the performances between theoretical WiMAX systems and real-world deployed systems. They have suggested that MIMO technique and the Space-Time Coding (STC) should be used in IEEE 802.16d to improve its performance. But, in some work [23] the authors argued that MIMO may be an inconvenient solution because of the limitation of volume and energy. They also claimed that cooperation among nodes is required to solve the problem. The authors have proposed a STC scheme with cooperative technology. Compared with MIMO STC scheme in mesh mode, this paper has demonstratedthat the use of cooperative STC scheme improves the system performance. The research work presented in [24] is similar to our work. In [24] the 
authors have investigated several modulation techniques for IEEE802.16d system including BPSK, QPSK, 16QAM and 64QAM. Different cyclic prefixes have been used for SUI-1 and SUI-2 channel conditions with channel bandwidth of $1.75 \mathrm{MHz}$. The authors have concluded that under a poor channel conditions QPSK modulation with 0.25 cyclic prefix is the best candidate because it provides the least BER. On the other hand, for good channel conditions the authors have suggested 16-QAM or 64-QAM.

Like all the mentioned related works the performances of IEEE 802.16d have been investigated in this work. Four different types of modulation scheme have been considered in this work namely BPSK, QPSK, 16-QAM, and 64-QAM. We considered all six SUI channel models namely SUI-1, SUI-2, SUI-3, SUI-4, SUI-5, and SUI-6 in this work contrasting the work in [24], where the authors have considered only two SUI channel models namely SUI-1 and SUI-2. Moreover, the main focus of the work presented in [24] is to find an appropriate combination of modulation and cyclic prefix for IEEE 802.16d system. But, our main focus is to investigate the effects of coding on the system performance under different SUI channel conditions.

\section{The Sui ChanNeL}

To investigate the performances of IEEE 802.16da practical wireless channel model is required. A practical wireless channel should be characterized by path loss, multipath delay spread,fading characteristics, Doppler spread, and interferences (co-channel and adjacent channel). Many practical channel models have been proposed in the literatures. Rayleigh fading channel and Ricean fading are two of them. In Rayleigh fading channel multipath propagation has been considered. In Ricean fading channel a LOS path has been included in addition to multipath propagation. In this channel model a narrow band received signal fading has been characterized by a Ricean distribution. The key parameter of this distribution is the $K$-factor, which is defined as the ratio of the LOS component power and the "scatter" multipath component power. In [4], an empirical model for $K$ factor has been derived from an experimental data collected in typical suburban environments for transmitter antenna heights of approximately $20 \mathrm{~m}$. The model presented therein is as follows:

$K=F_{s} F_{h} F_{b} K_{0} d \gamma u$

,where $F_{s}$ is a seasonal factor, $\mathrm{F}_{\mathrm{h}}$ is the receiving antenna height factor, $F_{b}$ is the beam width factor, $K_{o}$ and $\gamma$ are the regression coefficients, $u$ is a lognormal variable with mean of $0 \mathrm{~dB}$ and a standard deviation of $8.0 \mathrm{~dB}$. Some typical values of the parameters are $K_{o}=10$, and $\gamma=-0.5$, $F_{s}=1.0$ and $F_{s}=2.5$ in summer and winter respectively. The receiving antenna height factor $F_{h}$ is defined by $\mathrm{F}_{\mathrm{h}}=0.46(\mathrm{~h} / 3)$, where $h$ is the receiving antenna height in meters, the beam width factor $\mathrm{F}_{\mathrm{b}}$ is defined by $\mathrm{F}_{\mathrm{b}}=(\mathrm{b} / 17)-0.62$, where $b$ is in degrees. This empirical model has been confirmed by an experimental work presented in [5]. It has been shown that the experimental data closely matches with the model presented in [4]. The narrow band $K$-factor distribution was found to be lognormalwith the median as a simple function of season, antenna height, antenna beam width, and distance.

Channel models described above provide the basis for specifying the channel model for a given scenario. It is obvious that there are many possible combinations of parameters to obtain such channel specifications. A set of 6 typical channels was selected for three terrain types in SUI model.These three different types of terrain are shown in Table 3. Terrain C consisting of flat and light tree density and hence the effects of the obstruction are low in this case. SUI-1 and SUI-2 channels consider this type of terrain. The terrain B is characterized by flat/moderate tree density. SUI-3 and SUI-4 consider this type of terrain. The terrain A is characterized by a hilly area 
International Journal of Computer Networks \& Communications (IJCNC) Vol.6, No.2, March 2014

containing moderate to heavy tree density. SUI-5 and SUI-6 have been proposed for this type of terrain.

Table 3: Terrain of SUI model

\begin{tabular}{|l|l|l|}
\hline Terrain & Environmental Description & SUI Model \\
\hline C & Flat/Light Tree Density & SUI-1, SUI-2 \\
\hline B & Flat/Moderate Tree Density & SUI-3, SUI-4 \\
\hline A & Hilly/Moderate to Heavy Tree Density & SUI-5, SUI-6 \\
\hline
\end{tabular}

Table 4: SUI channel with low K-factor

\begin{tabular}{|c|c|c|c|}
\hline Doppler & Low Delay Spread & $\begin{array}{c}\text { Moderate Delay } \\
\text { Spread }\end{array}$ & $\begin{array}{c}\text { High Delay } \\
\text { Spread }\end{array}$ \\
\hline Low & SUI-3 & & SUI-5 \\
\hline High & & SUI-4 & SUI-6 \\
\hline
\end{tabular}

Table 5: SUI channel with high K-factor

\begin{tabular}{|c|c|c|c|}
\hline Doppler & Low Delay Spread & $\begin{array}{c}\text { Moderate Delay } \\
\text { Spread }\end{array}$ & $\begin{array}{c}\text { High Delay } \\
\text { Spread }\end{array}$ \\
\hline Low & SUI-1, SUI-2 & & SUI-5 \\
\hline High & & SUI-3, SUI-4 & \\
\hline
\end{tabular}

Table 4 and Table 5 depict that SUI-1 and SUI 2 have low delay spread because these two channels represent the terrain type $\mathrm{C}$, which has low tree density. The other terrain types have moderate to high delay spread. All these affect the $K$ factor of the channel. The SUI channels can also be classified as two more categories namely SUI channel with a low $K$-factor and SUI channel with high $K$-factor as shown in Table 4 and Table 5 respectively.

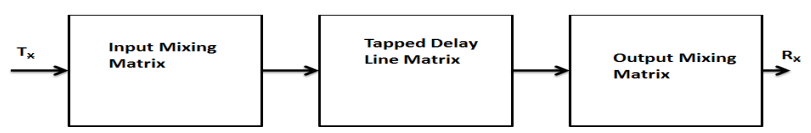

Figure 4: Transmitter and receiver block diagram for SUI channel

The general structure of SUI channel model is shown in Figure 4. The structure is general for any MIMO channels.Multi-Input-Multi-Output (MIMO) channel models have drawn considerable attentions. In MIMO system multiple antennas are used in the transmitter and/or in the receiver, which is a natural extension of the developments in antenna array based communication systems. The advantages of multiple receive antennas, such as gain and spatial diversity, have been wellknown and exploited in some research works $[6,7,8]$. The use of transmit diversity has also been investigated in some recent works $[9,10]$. The advantages of MIMO communication that exploits the physical channel between many transmit and receive antennas are currently receiving significant attentions [11]. The basic components of the SUI channel models are (a) input mixing matrix, (b) tapped delay line, and (c) output mixing matrix as shown in Figure 4.

The input mixing matrix models the correlation between input signals from multiple transmitting antennas. The tapped delay line matrixmodels the multipath fading of the channel. The multipath fading is modeled as a tapped-delay line with 3 taps with non-uniform delays. The gain associated with each tap is characterized by a Ricean distribution with a $K$-factor greater than zero or 
International Journal of Computer Networks \& Communications (IJCNC) Vol.6, No.2, March 2014

Rayleigh distribution with the $K$-factor equal to 0.The output mixing matrix models the correlation among the output signals for multiple receiving antennas.

Using the above general structure six SUI channels are have been proposed with the following parameters (i) cell size: $7 \mathrm{~km}$, (ii) BTS antenna height: $30 \mathrm{~m}$,(iii )receive antenna height: $6 \mathrm{~m}$, (iv) BTS antenna beam width: $120^{\circ}$, (v) receive antenna beam width: omni-directional $\left(360^{\circ}\right)$ and directional $\left(30^{\circ}\right)$, (vi) verticalpolarization only, and (vii) ninety percent cell coverage.For a $30^{\circ}$ antenna beam width 2.3 times smaller RMS delay spread is used compared to an omni-directional antenna[12]. Consequently, the second tap power is attenuated by an additional of $6 \mathrm{~dB}$ and the third tap power is attenuated by an addition of $12 \mathrm{~dB}$. For the omni-directional receive antenna case, the tap delay and the power settings are consistent with the COST 207 delay profile models.

Table 6: Characteristic of SUI-1 Channel

\begin{tabular}{|l|l|l|l|}
\hline Parameter & Tap 1 & Tap 2 & Tap 3 \\
\hline Delay $(\mu \mathrm{S})$ & 0 & 0.4 & 0.9 \\
\hline $\begin{array}{l}\text { Power (Omni-directional Antenna) } \\
(\mathrm{dB})\end{array}$ & 0 & -15 & -20 \\
90\% K-factor (Omni-directional) & 4 & 0 & 0 \\
$75 \% \mathrm{~K}-$ factor(Omni-directional) & 20 & 0 & 0 \\
\hline $\begin{array}{l}\text { Power }\left(30^{\circ} \text { Antenna) }(\mathrm{dB})\right. \\
90 \% \mathrm{~K}-\text { factor }\left(30^{\circ}\right)\end{array}$ & 0 & -21 & -32 \\
$75 \% \mathrm{~K}-$ factor $\left(30^{\circ}\right)$ & 16 & 0 & 0 \\
\hline Doppler Shift $(\mathrm{Hz})$ & 72 & 0 & 0 \\
\hline
\end{tabular}

In Table 6 SUI- 1 channel parameters have been listed. In this type of channel model the terrain used is low tree density hence the tap delay is low. There have been two types of antenna taken into account namely omni-directional and directional antenna. In both the cases there are two different values of the $K$-factor have been considered. For this type of channel the antenna correlation is 0.7 , which results in the reduction of the channel capacity [14].

Table 7: Characteristics of SUI-2 Channel

\begin{tabular}{|l|l|l|l|}
\hline Parameter & Tap 1 & Tap 2 & Tap 3 \\
\hline Delay $(\mu \mathrm{S})$ & 0 & 0.4 & 1.1 \\
\hline $\begin{array}{l}\text { Power (Omni-directional Antenna) } \\
(\mathrm{dB})\end{array}$ & 0 & -12 & -15 \\
$90 \%$ K-factor (Omni-directional) & 2 & 0 & 0 \\
$75 \%$ K-factor(Omni-directional) & 11 & 0 & 0 \\
\hline $\begin{array}{l}\text { Power }\left(30^{\circ} \text { Antenna) }(\mathrm{dB})\right. \\
90 \% \mathrm{~K}-\text { factor }\left(30^{\circ}\right)\end{array}$ & 0 & -18 & -27 \\
$75 \% \mathrm{~K}-f a c t o r\left(30^{\circ}\right)$ & 8 & 0 & 0 \\
\hline Doppler Shift $(\mathrm{Hz})$ & 36 & 0 & 0 \\
\hline
\end{tabular}

The other parameters of SUI-1 channel are antenna correlation and the $K$-factor. The value of antenna correlation under 0.5 has very negligible effect on the system capacity. The $K$-factor in this case is determined as 14 and 44.2 for directional antenna and omni-directional cases respectively. Table 7 shows the SUI-2 channel parameters. Compared to SUI-1 channel SUI-2 channel has higher delay for Tap 3. The antenna correlation is less than that of SUI- 1 channel. The SUI-2 channel has an antenna correlation of 0.5 , which has almost no effect on the model. The $K$-factor for omni-directional antenna is the same as SUI-1, but in case of directional antenna the K-factor reduces considerably. 
International Journal of Computer Networks \& Communications (IJCNC) Vol.6, No.2, March 2014

Table 8: Characteristics of SUI-3 Channel

\begin{tabular}{|l|l|l|l|}
\hline Parameter & Tap 1 & Tap 2 & Tap 3 \\
\hline Delay $(\mu \mathrm{S})$ & 0 & 0.4 & 0.9 \\
\hline $\begin{array}{l}\text { Power (Omni-directional Antenna) } \\
(\mathrm{dB})\end{array}$ & 0 & -5 & -10 \\
$90 \%$ K-factor (Omni-directional) & 1 & 0 & 0 \\
$75 \%$ K-factor(Omni-directional) & 7 & 0 & 0 \\
\hline Power $\left(30^{\circ}\right.$ Antenna) $(\mathrm{dB})$ & 0 & -11 & -22 \\
$90 \% \mathrm{~K}-$ factor $\left(30^{\circ}\right)$ & 3 & 0 & 0 \\
$75 \%$ K-factor $\left(30^{\circ}\right)$ & 19 & 0 & 0 \\
\hline Doppler Shift $(\mathrm{Hz})$ & 0.4 & 0.3 & 0.5 \\
\hline
\end{tabular}

Table 8shows the characteristics of SUI-3 channel. The tap delay in this case is more than SUI-2 and SUI-3 channel models. The system is considered again with anomni-directional antenna and a directional antenna with the $K$-factor of 0.5 and 1.6 respectively.The characteristics of the SUI- 4 channel model have been listed in Table 9. The SUI-4 has a low antenna correlation,

Table 9: Characteristic of SUI-4

\begin{tabular}{|l|l|l|l|}
\hline Parameter & Tap 1 & Tap 2 & Tap 3 \\
\hline Delay $(\mu \mathrm{S})$ & 0 & 1.5 & 4 \\
\hline Power (Omni-directional Antenna) & 0 & -4 & -8 \\
$(\mathrm{~dB})$ & 0 & 0 & 0 \\
$90 \%$ K-factor (Omni-directional) & 1 & 0 & 0 \\
$75 \%$ K-factor(Omni-directional) & & & \\
\hline $\begin{array}{l}\text { Power }\left(30^{\circ} \text { Antenna) }(\mathrm{dB})\right. \\
90 \% \mathrm{~K}-\text { factor }\left(30^{\circ}\right)\end{array}$ & 0 & -10 & -20 \\
$75 \% \mathrm{~K}-$ factor $\left(30^{\circ}\right)$ & 1 & 0 & 0 \\
\hline Doppler Shift $(\mathrm{Hz})$ & 5 & 0 & 0 \\
\hline
\end{tabular}

Table 10: Charactaristics of SUI-5

\begin{tabular}{|l|l|l|l|}
\hline Parameter & Tap 1 & Tap 2 & Tap 3 \\
\hline Delay $(\mu \mathrm{S})$ & 0 & 4.0 & 10 \\
\hline Power (Omni-directional Antenna) & 0 & -5 & -10 \\
$(\mathrm{~dB})$ & 0 & 0 & 0 \\
$90 \%$ K-factor (Omni-directional) & 0 & 0 & 0 \\
$75 \%$ K-factor(Omni-directional) & 2 & 0 & 0 \\
$50 \%$ K-factor (omni-directional) & & & \\
\hline Power $\left(30^{\circ}\right.$ Antenna) $(\mathrm{dB})$ & 0 & -11 & -22 \\
$90 \% \mathrm{~K}-$-factor $\left(30^{\circ}\right)$ & 0 & 0 & 0 \\
$75 \%$ K-factor(30 $)$ & 2 & 0 & 0 \\
$50 \%$ K-factor $\left(30^{\circ}\right)$ & 7 & 0 & 0 \\
\hline Doppler Shift $(\mathrm{Hz})$ & 0.2 & 0.15 & 0.25 \\
\hline
\end{tabular}


International Journal of Computer Networks \& Communications (IJCNC) Vol.6, No.2, March 2014

Table 11: Charactaristics of SUI-6

\begin{tabular}{|l|l|l|l|}
\hline Parameter & Tap 1 & Tap 2 & Tap 3 \\
\hline Delay $(\mu \mathrm{S})$ & 0 & 14.0 & 20 \\
\hline Power (Omni-directional Antenna) & 0 & -10 & -14 \\
$(\mathrm{~dB})$ & 0 & 0 & 0 \\
90\% K-factor (Omni-directional) & 0 & 0 & 0 \\
$75 \% \mathrm{~K}-$ factor(Omni-directional) & 1 & 0 & 0 \\
$50 \% \mathrm{~K}-$ factor (omni-directional) & & & \\
\hline Power $\left(30^{\circ}\right.$ Antenna) $(\mathrm{dB})$ & 0 & -16 & -26 \\
$90 \% \mathrm{~K}-$ factor $\left(30^{\circ}\right)$ & 0 & 0 & 0 \\
$75 \% \mathrm{~K}-f a c t o r\left(30^{\circ}\right)$ & 2 & 0 & 0 \\
$50 \% \mathrm{~K}-$ factor $\left(30^{\circ}\right)$ & 5 & 0 & 0 \\
\hline Doppler Shift $(\mathrm{Hz})$ & 0.4 & 0.3 & 0.5 \\
\hline
\end{tabular}

but the delays of different taps are more in this model. In omni-directional antenna case the delay is $1.257 \mu \mathrm{s}$ and for the directional case the delay is $0.563 \mu \mathrm{s}$. The $K$-factor is further reduced in this channel model. The $K$-factors are 0.2 and 0.6 in omni-directional. The $K$-factors are 1.0 and 3.2 for directional antenna. Table 10 and Table 11 show the characteristics of SUI-5 and SUI-6 channel models. In these models the antenna corellation is the same as the previous three models. But, the tap delay is less in SUI-5 channel as compared to SUI-6 channel. The $K$ - factors for both SUI-5 and SUI-6 channel models are identical.

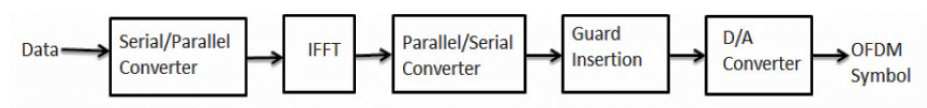

(a) Transmitter model

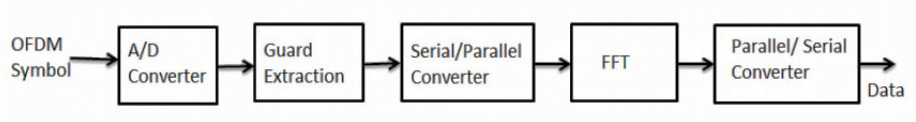

(a) Receiver

Figure 5: OFDM transmitter and receiver in SUI Channel

\section{SIMULATION MODELS AND RESUlTS}

In the simulation models we included three main components namely transmitter, receiver, and channel as shown in Figure 5. These components correspond to the physical layer of the IEEE 802.16d Wireless MAN OFDM air interface. Figure 5(a) shows the architecture of an OFDM transmitter. In this architecture the OFDM system treats the source symbols at the transmitter to be in the frequency domain. These symbols are used as the input to the IFFT block that brings the signal into the time domain. There are a set of sub-carriers (i.e., N), which are orthogonal with each other. Each of the $\mathrm{N}$ input orthogonal sub-carriers for the IFFT has a different frequency. The IFFT block modulates the $\mathrm{N}$ sinusoids onto $\mathrm{N}$ orthogonal subcarriers and provides an OFDM symbol. To overcome the interference of adjacent OFDM symbols cyclic prefix is inserted. IEEE $802.16 \mathrm{~d}$ allows the insertion of cyclic prefix of various lengths such as 1/4, 1/8, 1/16, and 1/32. The length of the cyclic prefix must be appropriately chosen so that they are longer than the maximum delay spread of the considered multipath environment.Then the Digital-to-Analog (D/A)converter converts OFDM symbol into an analog signal. Then this time-domain signal is 
transmitted across the channel. The OFDM receiver is illustrated in Figure 5(b). At the receiving end antenna receives an independent copy of the transmitted signal. Then an A/D converts that signal into digital one and cyclic prefix is removed as well. After passing through the FFT block the received signal is transformed into the frequency domain.

We have implemented and conducted the simulations in MatLab. The main program contains initialization parameters and input data. The parameters that can be set at the time of initialization are the number of simulated OFDM symbols, CP length, modulation, the range of SNR values, and SUI channel model for simulation. Some of the simulation parameters are listed in Table 12. The performances of different modulation schemes namely BPSK, QPSK, 16-QAM, and 64QAM under different SUI channel models are shown in Figure 6, Figure 7,Figure 8, and Figure 9 respectively. In all modulation schemes two cases have been considered namely coded system and uncoded system. The simulation results of BPSK are shown in Figure 6. It is shown that the coded system outperforms the uncoded system at a very low $\mathrm{E}_{\mathrm{b}} / \mathrm{N}_{0}$ (around $6 \mathrm{~dB}$ ) irrespective of considered SUI channel model

Table 12: Simulation parameters

\begin{tabular}{|c|c|}
\hline Parameter & Value \\
\hline No. of Symbols & 128 \\
\hline Bandwidth & $1.25 \mathrm{MHz}$ \\
\hline Cyclic Prefix & $1 / 16$ \\
\hline Modulation & BPSK, QPSK,16-QAM,64-QAM \\
\hline Channels & SUI-1,SUI-2,SUI-3,SUI-4,SUI-5, SUI-6 \\
\hline Coding & Without coding, \\
& With Coding (Reed-Solomon, Convolutional) \\
\hline
\end{tabular}

The simulation results for QPSK modulation are shown in Figure 7. The simulation results show that the performance of QPSK is similar to that of BPSK modulation under different SUI channel conditions. Only exceptions occurred at SUI-5and SUI-6 channel models. In these two models the coded QPSK needed a higher $\mathrm{E}_{\mathrm{b}} / \mathrm{N}_{\mathrm{o}}$ (i.e., $10 \mathrm{~dB}$ ) to outperform the uncoded QPSK system. In addition, the coded and uncoded QPSK systems perform almost in the same way for the SUI-6 channel model.

The performances of the coded and the uncoded 16-QAM systems under different SUI channel models are shown in Figure 8. In contrast to BPSK and QPSK systems the simulations results for 16-QAM show that a higher $\mathrm{E}_{\mathrm{b}} / \mathrm{N}_{0}$ is required for the coded system to outperform the uncoded system for SUI-1, SUI-2, SUI-3, and SUI-4 channel models. There is no significant performance difference for the SUI-5 and SUI-6 channel models. Under SUI-5 channel model the performance improvement of the coded system over the uncoded system is more a less same. Under the SUI-6 channel condition the coded system even performs poorly compared to its uncoded counterpart. The results for the SUI- 6 channel model show that even a high $\mathrm{E}_{\mathrm{b}} / \mathrm{N}_{\mathrm{o}}$ cannot help a coded system to outperform the uncoded system.

Finally, the performances of the coded and uncoded 64-QAM modulation system under different SUI channel conditions are illustrated in Figure 9. The simulation results show that a high $\mathrm{E}_{\mathrm{b}} / \mathrm{N}_{0}$ is required for the coded system to outperform the uncoded system under all SUI channel conditions. In some cases like SUI-3 and SUI-5 channels the uncoded system even outperforms a coded system. 
International Journal of Computer Networks \& Communications (IJCNC) Vol.6, No.2, March 2014

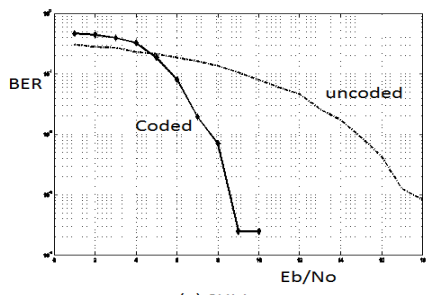

(a) SUI-I

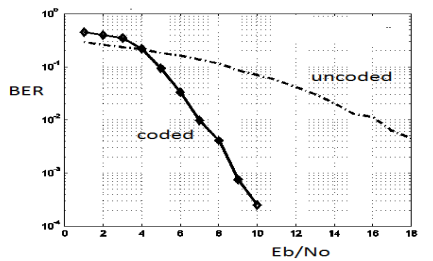

(c) SUI-3

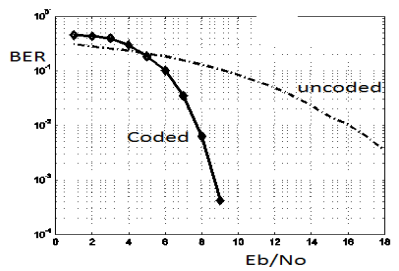

(e) SUI-5

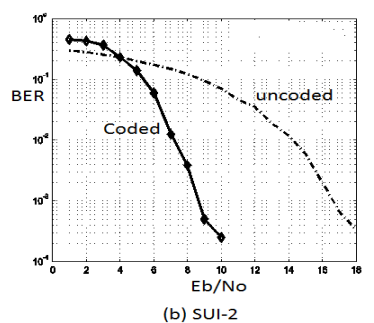

(b) SUI-2

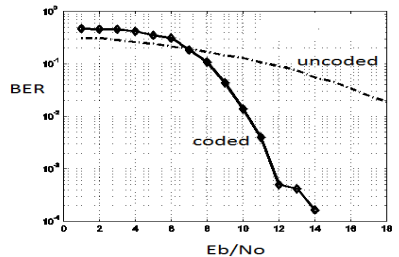

(4) SUI-3

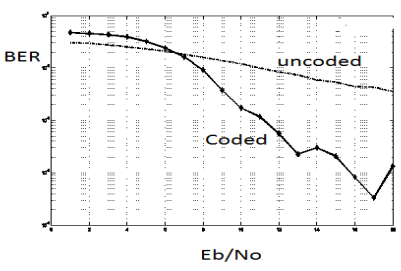

(f) SUI-6

Figure 6: Performances of BPSK for different SUI channels
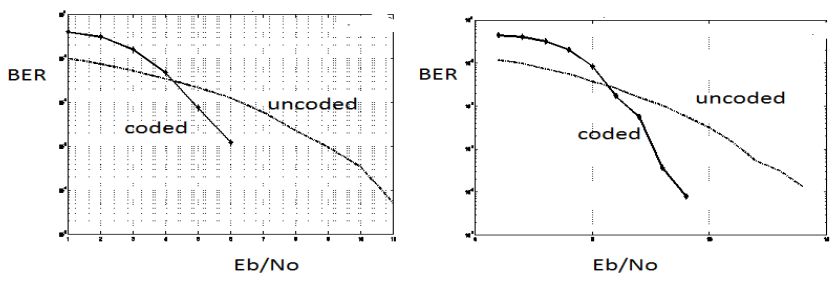

(a) SUI-1

(a) SUI-2
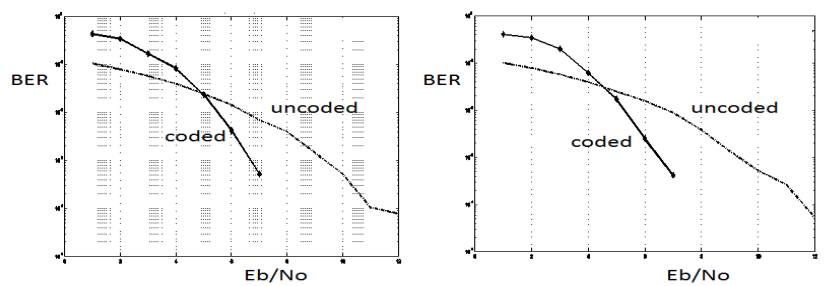

(c) SUI-3

(c) SUI-4
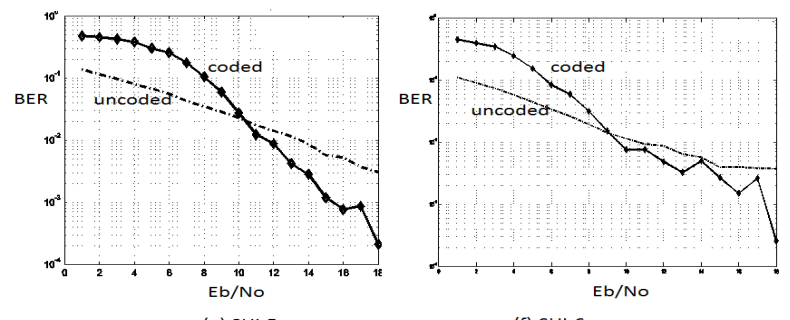

(f) SUI-6

Figure 7: Performances of QPSK for different SUI channels 
International Journal of Computer Networks \& Communications (IJCNC) Vol.6, No.2, March 2014
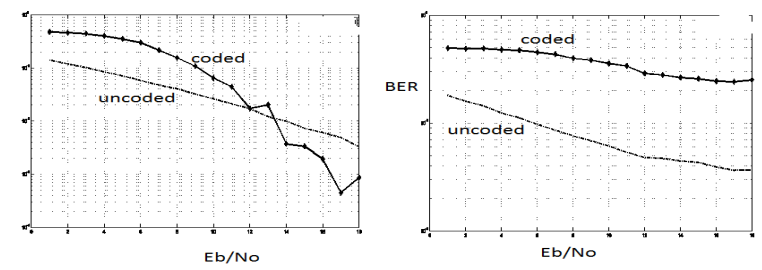

(e) SUI-5
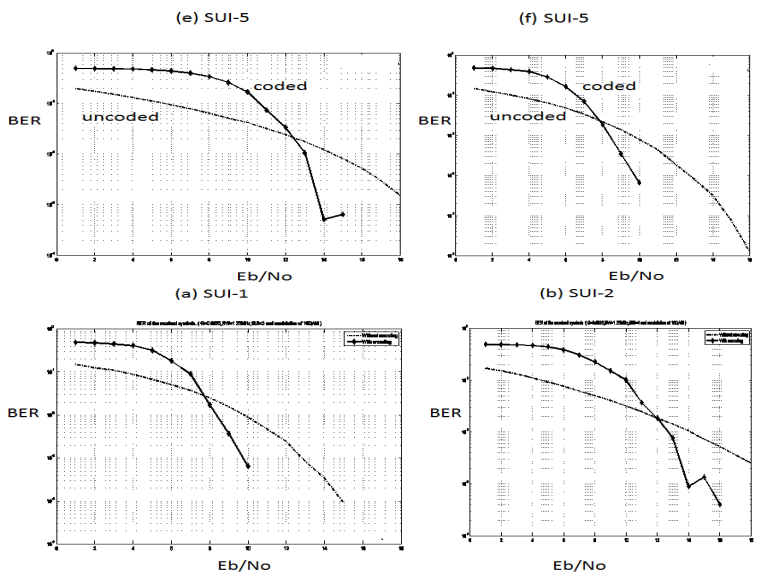

(c) sul-3

(d) sul-4

Figure 8: Performances of 16-QAM for different SUI channels
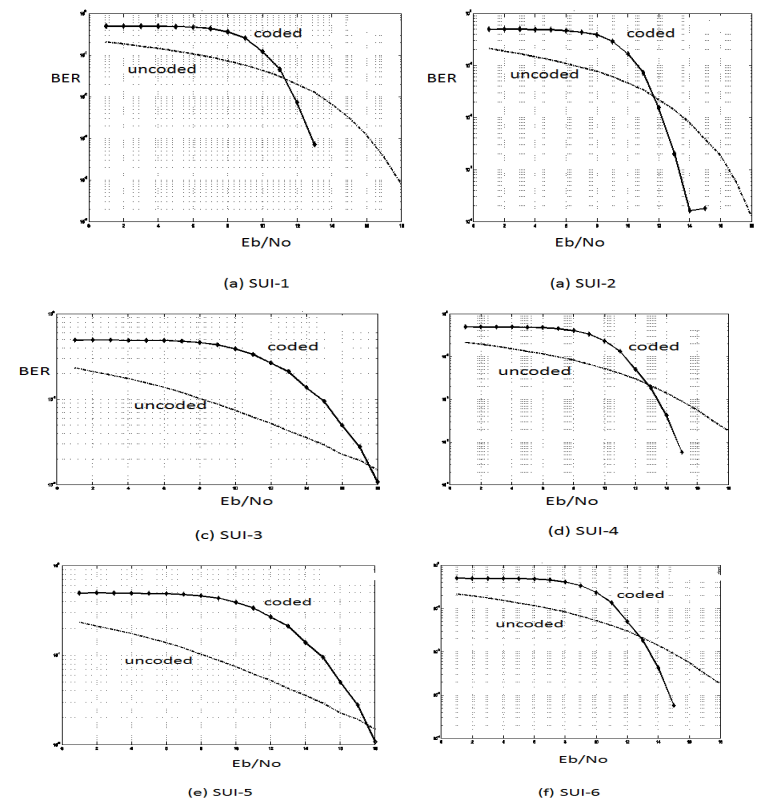

Figure 9: Performances of 64-QAM for different SUI channels

\section{Conclusions}

In this paper the IEEE $802.16 \mathrm{~d}$ physical layer has been investigated under different modulation schemes and also under different SUI channel conditions. Both coded and uncoded systems have been investigated in this paper. In general the simulation results show that a coded system 
outperforms an uncoded system above a certain level of $\mathrm{E}_{\mathrm{b}} / \mathrm{N}_{0}$. But, this level depends on the modulation scheme used and the channel conditions. It has also been shown that a coded system may not outperform an uncoded system even at a very high $\mathrm{E}_{\mathrm{b}} / \mathrm{N}_{0}$. For example, 16-QAM and 64QAM do not show any improvement in coded system compared to an uncoded system. But, BPSK and QPSK modulation schemes show that a coded system is preferred to an uncoded system. Hence, a coded IEEE 802.16 system may not help always under all channel conditions. In some channel conditions, an uncoded system can be used and the system complexity can be reduced.

In this work we investigated the performances based on the modulations like BPSK, QPSK,16QAM, and 64-QAM. In future, this investigation can be extended to include more sophisticated modulation techniques like BFSK, MSK, and GMSK. In addition, more advance coding technique like Turbo coding can also be included in the future investigations, which is also an optional feature of IEEE 802.16d.

\section{REFERENCES}

[1] Theodore S. Rappaport, "Wireless Communication: Principles and Practices", Prentice Hall, upper Saddle River, New Jersey, $2^{\text {nd }}$ Edition, pp.176-190

[2] Young Soo Cho and JaeK Wan Kim, Won Young Yang and Chung Goo Kang, “ MIMO OFDM Wireless Communication in MatLab, IEEE Press and John Wiley, pp.25-68

[3] www.Wirelessman.org

[4] L.J. Greenstein, S. Ghassemzadeh, V.Erceg, and D.G. Michelson, "Ricean K-factors in narrow band fixed wirelesschannels: Theory, experiments, and statistical models," In the Proceedings ofWPMC'99, Amsterdam, September 1999.

[5] D.S. Baum et.al., "Measurements and characterization of broadband MIMO fixed wireless channels at 2.5 GHz", In the Proceedings of ICPWC'2000, Hyderabad, December 2000.

[6] W.C. Jakes, “Microwave Mobile Communications”, John Wiley, New York, 1974.

[7] R.A. Monzingo and T.W. Miller, "Introduction to Adaptive Arrays”, JohnWiley, New York, 1980.

[8] K.W. Forsythe, D.W. Bliss, and C.M. Keller, "Multichannel Adaptive Beamforming and InterferenceMitigation in Multiuser CDMA Systems," In the proceedings of the thirty-third Asilomar Conference on Signals, Systems \& Computers 1, Pacific Grove, California, 24-27

October, 1999, pp.506-510.

[9] A. Wittneben, "Basestation Modulation Diversity for Digital SIMULCAST",In the proceedings of IEEE Vehicular Technology Conference, St. Louis, May 1991, pp. 848-853.

[10] V. Weerackody, "Diversity for Direct-Sequence Spread Spectrum Using Multiple Transmit Antennas," In the proceedings of IEEE International Communications Conference, Geneva,

23-26 May, 1993, pp. 1775-1779.

[11] "Advantages and Disadvantages of OFDM available at http://sna.csie.ndhu.edu.tw/ cnyang/MCCDMA/tsld021.htm

[12] B.M. Hochwald and W. Sweldens, "Differential Unitary Space-Time Modulation," IEEE Transaction on Communication Vol. 48, No. 12, 2000, pp. 45-48

[13] Bernard Skalar, "Digital Communications: Fundamentals and Applications”, Second Edition, Pearson Education Inc, 2001, pp. 323-328.

[14] Ge, Yiqun, Shi Waxian, and Sun Goubin, “ A Study of Iterative Joint Sunchronization for Time 
Offset and Frequency Offset in IEEE 802.16d WirelessMAN OFDM System”, Proceedings of the $5^{\text {th }}$ International Conference on Information, Communications, and Signal Processing, Bankok, Thailand, 2005, pp. 1217-1221

[15] Leung. K.K. and Mukharjee, S. and Rittenhouse, G.E., "Mobility Support for IEEE 802.16d Wireless Network", Proceedings of IEEE Wireless Communications and Networking

Conference, March 2005, Vol. 3, pp. 1446 - 1452

[16] A. Gosh, D.R. Wolter, and J.G. Andrews, "Broadband Wireless Access with WiMax/802.16:Current Performance Benchmarks and Future Potential”, IEEE Communication Magazine, February 2005, pp. 129-136

[17] Young, S.S. and D. S. Kwon, “Uplink SDMA Performance Comparison of Single and Multiple Antenna Mobiles", Proceedings of the $66^{\text {th }}$ IEEE Vehicular Technology Conference, SeptemberOctober, 2007, Baltimore, MD, pp. 676-679

[18] Pei Xiao, Barbero, L.G., Sallathural, M., and Ratnaraj, T.,” On the Uncoded BER Performance Bound of the IEEE 802.16d Channel", IEEE Signal Processing Letter, Vol. 15. October 2008, pp. $561-564$

[19] Hosseinnezhad, M., and Salahi, A., " Low Complexity MMSE based Channel Estimation Algorithm for the IEEE 802.16d WMAN Standard", Proceedings of the International Symposium on Telecommunications, Tehran, August 2008, pp. 257-262

[20] Xinbo, Jin, Pingam Li, Jiaghai Wang, “ The Research of Rotated Quasi-Orthogonal Space-Time Block Codes for OFDM Communications Based on IEEE 802.16d", Proceedings of the $4^{\text {th }}$ International Conference on Wireless Communications, Networking, and Mobile Computing, October 2008, Dalian, pp. 1-4

[21] Aldhaheri, R.W., and Al-Qahtani, A.H., " Performance Analysis of fixed and mobile WiMax MCCDMA-based System", Proceedings of the $7^{\text {th }}$ International Symposium on Wireless Communication Systems, York, September 2010, pp. 436-440

[22] Martin, J., Bo Li, Pressly, W., Westall, J., “ WiMax Performance at 4.9 GHz”, Proceedings of IEEE Aerospace Conference, Big Sky, MT, March 2010, pp. 1-8

[23] Jing Shi, Xu Li, Xuezhen, and Zhang, ChengChen, “ A Cooperative STC Scheme for IEEE 802.16d", Proceedings of the International Conference in Information Theory and Information Security, Beijing, December 2010, pp. 969-972

[24] Sekar, U., Verrapagounder, P., Baskaran, B., “ BER performance and bandwidth efficiency analysis of fixed WiMax", Proceedings of the third International Conference on Computing Communication and Networking Technologies, Coimbatore, July 2012, pp. 1-4 